\title{
INEQUALITIES FOR SYMMETRIC FUNCTIONS AND HERMITIAN MATRICES
}

\author{
M. MARCUS AND L. LOPES
}

1. Introduction. The purpose of this paper is to present two concavity results for symmetric functions and apply these to obtain inequalities connecting the characteristic roots of the non-negative Hermitian (n.n.h.) matrices $A, B$ and $A+B$.

H. F. Bohnenblust has recently communicated to one of the present authors a suggestion of $\mathrm{H}$. Samelson indicating that Theorem 2 below can be derived from a concavity result on mixed volumes of convex bodies due to Fenchel (2). Bohnenblust has also obtained a separate proof of Theorem 2 by different methods and we are indebted to him for several valuable conversations concerning his results. Theorem 1 in the sequel is believed to be new and we show that it actually provides a simple and direct algebraic proof of Theorem 2 without using the results on mixed volumes. We have not been able to find an explicit statement of Theorem 2 in the literature. Both the present proof and the unpublished proof of Bohnenblust were constructed before Samelson observed the connection with Fenchel's result.

In $\$ 2$ we state and prove the two results on symmetric functions. In $\$ 3$ these results are applied to obtain inequalities connecting the characteristic roots of n.n.h. matrices. Theorem 4 extends the classical Minkowski determinant inequality to all of the coefficients in the characteristic polynomial and Theorem 5 extends a recent concavity result of Fan (1) for determinants.

2. Results on Symmetric Functions. Let $(a)=\left(a_{1}, \ldots, a_{n}\right)$ be a set of real numbers and let $E_{r}(a)$ denote the $r$ th elementary symmetric function of $(a), 1 \leqslant r \leqslant n$ :

$$
E_{r}(a)=\sum_{1 \leqslant i_{1}<\ldots<i_{r} \leqslant n} \prod_{j=1}^{r} a_{i_{j}},
$$

where the summation extends over all $\left(\begin{array}{c}n \\ r\end{array}\right)$ choices of $i_{1}<\ldots<i_{r}$ from $1, \ldots, n$. If $r=0$ then $E_{r}(a)=1$. If $(a)=\left(a_{1}, \ldots, a_{n}\right)$ and $(b)=\left(b_{1}, \ldots, b_{n}\right)$ then let $(a+b)=\left(a_{1}+b_{1}, \ldots, a_{n}+b_{n}\right)$. We confine our attention here to sets $(a)$ of non-negative numbers at least $r$ of whose elements are assumed positive. If there exists a number $\lambda$ such that

$$
a_{j}=\lambda b_{j}, \quad j=1, \ldots, n,
$$

we shall write $(a) \sim(b)$. If this is not the case we indicate it by $(a) \nsim(b)$.

Received May 14, 1956. Part of this research was completed under U.S. Air Force contract AF 18(603)-83. 
The set $a_{1}, \ldots, a_{j-1}, a_{j+1}, \ldots, a_{n}$ will be denoted by $\left(a^{\prime}{ }_{j}\right)$. We shall also use the abbreviation

$$
\mathbf{E}_{r}(a)=\frac{E_{r}(a)}{E_{r-1}(a)}
$$

THEOREM 1. If $1 \leqslant r \leqslant n$ then

$$
\mathbf{E}_{r}(a+b)>\mathbf{E}_{r}(a)+\mathbf{E}_{r}(b)
$$

unless $(a) \sim(b)$ or $r=1$ in which cases we have equality.

Proof. We use an induction on $r$. If $(a) \sim(b)$ we clearly have equality, hence assume $(a) \nsim(b)$. For $r=2$ the result follows from

$$
\mathbf{E}_{2}(a+b)-\mathbf{E}_{2}(a)-\mathbf{E}_{2}(b)=\frac{\sum_{i=1}^{n}\left(a_{i} \sum_{j=1}^{n} b_{j}-b_{i} \sum_{j=1}^{n} a_{j}\right)^{2}}{2 \sum_{i=1}^{n}\left(a_{i}+b_{i}\right) \sum_{i=1}^{n} a_{i} \sum_{i=1}^{n} b_{i}}
$$

Hence assume $r>2$. We first note that

$$
\sum_{i=1}^{n} a_{i} E_{r-1}\left(a_{i}^{\prime}\right)=r E_{r}(a) .
$$

Now

$$
E_{r}(a)=a_{i} E_{r-1}\left(a^{\prime}{ }_{i}\right)+E_{\tau}\left(a_{i}^{\prime}\right),
$$

and summing on $i$ we have

$$
n E_{r}(a)=\sum_{i=1}^{n} a_{i} E_{r-1}\left(a^{\prime}{ }_{i}\right)+\sum_{i=1}^{n} E_{r}\left(a^{\prime}{ }_{i}\right) .
$$

Using (1) it follows that

$$
\sum_{i=1}^{n} E_{r}\left(a^{\prime}{ }_{i}\right)=(n-r) E_{r}(a) .
$$

Now write

$$
\begin{gathered}
E_{r}(a)-E_{r}\left(a^{\prime}{ }_{i}\right)=a_{i} E_{r-1}\left(a^{\prime}{ }_{i}\right) \\
=a_{i} E_{r-1}(a)-a_{i}^{2} E_{r-2}\left(a^{\prime}{ }_{i}\right)
\end{gathered}
$$

summing over $i$ and using (2) we have

$$
\begin{aligned}
r E_{r}(a) & =\sum_{i=1}^{n} a_{i} E_{r-1}(a)-\sum_{i=1}^{n} a_{i}^{2} E_{r-2}\left(a^{\prime}{ }_{i}\right), \\
\frac{E_{r}(a)}{E_{r-1}(a)} & =\frac{1}{r}\left\{\sum_{i=1}^{n} a_{i}-\sum_{i=1}^{n} a_{i}^{2} \frac{E_{r-2}\left(a_{i}^{\prime}\right)}{E_{r-1}(a)}\right\} \\
& =\frac{1}{r}\left\{\sum_{i=1}^{n} a_{i}-\sum_{i=1}^{n} \frac{a_{i}^{2}}{a_{i}+\mathbf{E}_{r-1}\left(a_{i}^{\prime}\right)}\right\} .
\end{aligned}
$$


From (3) we calculate that

$$
\begin{gathered}
\phi=\mathbf{E}_{r}(a+b)-\mathbf{E}_{r}(a)-\mathbf{E}_{r}(b) \\
=\frac{1}{r} \sum_{i=1}^{n}\left\{\frac{a_{i}^{2}}{a_{i}+\mathbf{E}_{r-1}\left(a^{\prime}{ }_{i}\right)}+\frac{b_{i}^{2}}{b_{i}+\mathbf{E}_{r-1}\left(b^{\prime}{ }_{i}\right)}-\frac{\left(a_{i}+b_{i}\right)^{2}}{a_{i}+b_{i}+\mathbf{E}_{r-1}\left(a^{\prime}{ }_{i}+b^{\prime}{ }_{i}\right)}\right\} .
\end{gathered}
$$

We make the induction hypothesis that the inequality holds for $r-1$. Since $r \geqslant 3$ we have

$$
\mathbf{E}_{r-1}\left(a^{\prime}{ }_{i}+b^{\prime}{ }_{i}\right)>\mathbf{E}_{r-1}\left(a^{\prime}{ }_{i}\right)+\mathbf{E}_{r-1}\left(b^{\prime}{ }_{i}\right)
$$

unless $\left(a^{\prime}{ }_{i}\right) \sim\left(b^{\prime}{ }_{i}\right)$ in which case we have equality. We first consider the case for which there exists an $i$ such that $\left(a^{\prime}{ }_{i}\right) \nsim\left(b^{\prime}{ }_{i}\right)$; then it follows that

$$
\begin{gathered}
\phi>\frac{1}{r} \sum_{i=1}^{n}\left\{\frac{a_{i}^{2}}{a_{i}+\mathbf{E}_{r-1}\left(a^{\prime}{ }_{i}\right)}+\frac{b_{i}^{2}}{b_{i}+\mathbf{E}_{r-1}\left(b^{\prime}{ }_{i}\right)}\right. \\
\left.-\frac{\left(a_{i}+b_{i}\right)^{2}}{a_{i}+b_{i}+\mathbf{E}_{r-1}\left(a^{\prime}{ }_{i}\right)+\mathbf{E}_{r-1}\left(b_{i}{ }_{i}\right)}\right\} \\
=\frac{1}{r} \sum_{i=1}^{n} \frac{\left(a_{i} \mathbf{E}_{r-1}\left(b^{\prime}{ }_{i}\right)-b_{i} \mathbf{E}_{r-1}\left(a^{\prime}{ }_{i}\right)\right)^{2}}{\left(a_{i}+\mathbf{E}_{r-1}\left(a^{\prime}{ }_{i}\right)\right)\left(b_{i}+\mathbf{E}_{r-1}\left(b^{\prime}{ }_{i}\right)\right)\left(a_{i}+b_{i}+\mathbf{E}_{r-1}\left(a_{i}^{\prime}\right)+\mathbf{E}_{r-1}\left(b^{\prime}{ }_{i}\right)\right)}
\end{gathered}
$$

Now in case $\left(a^{\prime}{ }_{i}\right) \sim\left(b^{\prime}{ }_{i}\right)$ for each $i$ then the first inequality in (4) is equality. To resolve this case suppose

$$
\left(a^{\prime}{ }_{i}\right)=\lambda_{i}\left(b^{\prime}{ }_{i}\right)
$$

for each $i$. Then

$$
\left(a_{i} \mathbf{E}_{r-1}\left(b^{\prime}{ }_{i}\right)-b_{i} \mathbf{E}_{r-1}\left(a^{\prime}{ }_{i}\right)\right)^{2}=\left(a_{i}-\lambda_{i} b_{i}\right)^{2} \omega_{i}^{2}
$$

where

$$
\omega_{i}=\mathbf{E}_{r-1}\left(b^{\prime}{ }_{i}\right) \neq 0
$$

since at least $r$ of the $(b)$ are positive. But on the other hand $a_{i}=\lambda_{i} b_{i}$ implies $(a) \sim(b)$ contrary to assumption.

Hence in this case the second inequality in (4) is strict and the induction is complete.

THEOREM 2. If $(a)$ and $(b)$ are sets of $n$ positive variables then for $1 \leqslant r \leqslant n$

$$
E_{r}^{1 / r}(a+b)>E_{r}^{1 / r}(a)+E_{r}^{1 / r}(b)
$$

unless $(a) \sim(b)$ or $r=1$, in which cases we have equality.

To prove (5) we first establish a preliminary

Lemma. If $\left(a_{n}^{\prime}\right) \sim\left(b_{n}^{\prime}\right)$ and $(a) \nsim(b)$ then the inequality (5) holds.

Proof. ${ }^{1}$ Set

$$
\begin{aligned}
t a_{i} & =b_{i}, \\
(t+\delta) a_{n} & =b_{n}, \quad \delta \neq 0 .
\end{aligned} \quad i=1, \ldots, n-1
$$

${ }^{1}$ The authors are indebted to K. Hoechsmann for simplifying the original proof of this Lemma. 
From

$$
E_{r}(b)=E_{r}\left(b_{n}^{\prime}\right)+b_{n} E_{r-1}\left(b^{\prime}{ }_{n}\right)
$$

we conclude that

$$
\begin{aligned}
E_{r}(b) & =t^{r} E_{r}\left(a_{n}^{\prime}\right)+(t+\delta) t^{r-1} a_{n} E_{r-1}\left(a_{n}^{\prime}\right) \\
& =t^{r} E_{r}(a)+\delta t^{r-1} a_{n} E_{r-1}\left(a_{n}^{\prime}\right) .
\end{aligned}
$$

Set

$$
\begin{aligned}
& g(\delta)=E_{r}^{1 / r}(a+b)-E_{r}{ }^{1 / r}(a)-E_{r}^{1 / r}(b) . \\
& g(\delta)=\left((t+1)^{r} E_{r}(a)+\delta(t+1)^{r-1} a_{n} E_{r-1}\left(a_{n}^{\prime}\right)\right)^{1 / r} \\
& -E_{r}^{1 / r}(a)-\left(t^{r} E_{r}(a)+\delta t^{r-1} a_{n} E_{r-1}\left(a_{n}^{\prime}\right)\right)^{1 / r} .
\end{aligned}
$$

Since $g(0)=0$ it will suffice to establish

$$
\delta g^{\prime}(\delta)>0
$$

for $\delta \neq 0$, for then $g$ strictly increases from $\delta=0$. First note that

$$
g^{\prime}(\delta)=\frac{a_{n} E_{r-1}\left(a_{n}\right)(t+1)^{r-1} E_{r}^{(r-1) / r}(b)-t^{r-1} E_{r}^{(r-1) / r}(a+b)}{r\left(E_{r}(b) E_{r}(a+b)\right)^{(r-1) / r}} .
$$

The sign of this expression is the same as the sign of

$$
\begin{aligned}
(t+1)^{r} E_{r}(b)-t^{r} E_{r}(a+b) & =(t+1)^{r} t^{r} E_{r}(a)+\delta t^{r-1} a_{n} E_{r-1}\left(a_{n}\right) \\
& -t^{r}(t+1)^{r} E_{r}(a)+\delta(t+1)^{r-1} a_{n} E_{r-1}\left(a_{n}\right) \\
& =t^{r-1}(t+1)^{r-1} \delta a_{n} E_{r-1}\left(a_{n}\right) .
\end{aligned}
$$

Hence $g^{\prime}(\delta)$ has the same sign as $\delta$.

The proof of (5) will be done by a double induction on $r$ and $n$. The case $r=2$ and $n$ arbitrary can easily be verified directly. We thus make the induction hypotheses that (5) holds for

(i) $r-1$ and all $n \geqslant r-1$,

(ii) $r$ and $n-1$.

Since we know $r=2$ and $r=n$ (Hölder's inequality) it will suffice to prove

(5) for $r, n$ using (i) and (ii).

By the Lemma we can assume $\left(a_{n}^{\prime}\right) \nsim\left(b_{n}^{\prime}\right)$ and also $(a) \nsim(b)$. Set

$$
x^{2}=a_{n}, \quad y^{2}=b_{n}, \quad x=R \cos \phi, \quad y=R \sin \phi .
$$

Let $f(x, y)=g(R, \phi)$ be the left side of (5) minus the right side:

$$
\begin{aligned}
g(R, \phi) & =\left(E_{r-1}\left(a_{n}^{\prime}+b^{\prime}{ }_{n}\right) R^{2}+E_{r}\left(a_{n}^{\prime}+b^{\prime}{ }_{n}\right)\right)^{1 / r} \\
& -\left(E_{r-1}\left(a_{n}^{\prime}\right) R^{2} \cos ^{2} \phi+E_{r}\left(a^{\prime}{ }_{n}\right)\right)^{1 / r} \\
& -\left(E_{r-1}\left(b^{\prime}{ }_{n}\right) R^{2} \sin ^{2} \phi+E_{r}\left(b_{n}^{\prime}{ }_{n}\right)\right)^{1 / r} .
\end{aligned}
$$

We show first that there exists a sufficiently large $R_{0}$ such that

$$
g(R, \phi)>0
$$


for $0 \leqslant \phi \leqslant 2 \pi$ and $R \geqslant R_{0}$. Set

$$
\begin{aligned}
& C_{j}=E_{j}\left(a_{n}^{\prime}+b_{n}^{\prime}\right), \\
& A_{j}=E_{j}\left(a_{n}^{\prime}\right), \\
& B_{j}=E_{j}\left(b_{n}^{\prime}\right) .
\end{aligned}
$$

By factoring an $R^{2}$ from each bracketed quantity in (6) it is clear that

$$
g(R, \phi)>0
$$

if and only if

$$
\left(C_{r-1}+\frac{C_{r}}{R^{2}}\right)^{1 / r}-\left(A_{r-1} \cos ^{2} \phi+\frac{A_{r}}{R^{2}}\right)^{1 / r}-\left(B_{r-1} \sin ^{2} \phi+\frac{B_{r}}{R^{2}}\right)^{1 / r}>0 .
$$

At $\phi=0$ and $\phi=2 \pi$ this is clear. By setting

$$
h^{\prime}(\phi)=0
$$

we easily calculate that at a minimum for $h(\phi)$

$$
\begin{aligned}
& \cos ^{2} \phi=A_{r-1}{ }^{1 /(r-1)}\left(A_{r-1}{ }^{1 /(r-1)}+B_{r-1}{ }^{1 /(r-1)}\right)^{-1}, \\
& \sin ^{2} \phi=B_{r-1}{ }^{1 /(r-1)}\left(A_{r-1}^{1 /(r-1)}+B_{r-1}^{1 /(r-1)}\right)^{-1} .
\end{aligned}
$$

Replacing these values in $h(\phi)$ and using (i) we see that

$$
h(\phi)>0
$$

and hence

$$
f(x, y)=g(R, \phi)>0
$$

for $R \geqslant R_{0}$. Thus if $f(x, y)$ is to have a non-positive minimum it must occur in the interior of the circle $R=R_{0}$ where the partials satisfy

$$
\begin{aligned}
& f_{x}=0 \\
& f_{y}=0 .
\end{aligned}
$$

The equations (7) become

$$
\begin{aligned}
& x\left\{\left(\left(x^{2}+y^{2}\right) C_{r-1}+C_{r}\right)^{(1-r) / r} C_{r-1}-\left(x^{2} A_{r-1}+A_{r}\right)^{(1-r) / r} A_{r-1}\right\}=0 \\
& y\left\{\left(\left(x^{2}+y^{2}\right) C_{r-1}+C_{r}\right)^{(1-r) / r} C_{r-1}-\left(y^{2} B_{r-1}+B_{r}\right)^{(1-r) / r} B_{r-1}\right\}=0 .
\end{aligned}
$$

If $x=y=0$ we have the result by (ii). If both the curly bracketed quantities in (8) vanish then the result follows easily from (i) by direct substitution. The less trivial case is, say, $y=0$ and $x \neq 0$. We must then show

$$
f(x, 0)>0
$$

for the value $x$ given by

$$
x^{2}=\frac{C_{r-1}^{1 /(r-1)} \frac{A_{r}}{A_{r-1}}-A_{r-1}^{1 /(r-1)} \frac{C_{r}}{C_{r-1}}}{A_{r-1}^{1 /(r-1)}-C_{r-1}^{1 /(r-1)}} .
$$


Replacing this in $f(x, 0)$ we have $f(x, 0)=$

$$
\begin{gathered}
\left(\frac{C_{r} C_{r-1}^{1 /(r-1)}-C_{r-1}^{r /(r-1)} \frac{A_{r}}{A_{r-1}}}{C_{r-1}^{1 /(r-1)}-A_{r-1}^{1 /(r-1)}}\right)^{1 / r}-\left(\frac{A_{r-1}^{r /(r-1)} \frac{C_{r}}{C_{r-1}}-A_{r} A_{r-1}^{1 /(r-1)}}{C_{r-1}^{1 /(r-1)}-A_{r-1}^{1 /(r-1)}}\right)^{1 / r}-B_{r}^{1 / r} \\
=C_{r-1}^{1 /(r-1)}\left(\frac{\frac{C_{r}}{C_{r-1}}-\frac{A_{r}}{A_{r-1}}}{C_{r-1}^{1 /(r-1)}-A_{r-1}^{1 /(r-1)}}\right)^{1 / r}-A_{r-1}^{1 /(r-1)}\left(\frac{\frac{C_{r}}{C_{r-1}}-\frac{A_{r}}{A_{r-1}}}{C_{r-1}^{1 /(r-1)}-A_{r-1}^{1 /(r-1)}}\right)^{1 / r}-B_{r}^{1 / r} .
\end{gathered}
$$

At this point we use the inequality of 'Theorem 1. Then

$$
f(x, 0)>\left(\frac{B_{r}}{B_{r-1}}\right)^{1 / r}\left(C_{r-1}^{1 /(r-1)}-A_{r-1}^{1 /(r-1)}\right)^{(r-1) / r}-B_{r}^{1 / r}>0 .
$$

The last inequality follows from (i). This completes the induction.

To indicate briefly that the concavity of $E_{r}{ }^{1 / r}(a)$ can be obtained from Fenchel's result we set $K_{i}$ equal to the segment from 0 to 1 on the $i$ th axis $(i=1, \ldots, n)$ and we let $Q$ be the unit cube in $n$-space. For $a=\left(a_{1}, \ldots, a_{n}\right)$, $a_{i} \geqslant 0$, form the convex sum

$$
K_{a}=\sum_{i=1}^{n} a_{i} K_{i}
$$

For $\lambda, \mu$ non-negative numbers form the volume

$$
V\left(\lambda K_{a}+\mu Q\right)=\sum_{j=0}^{n} b_{j} \lambda^{n-j} \mu^{j} .
$$

The coefficients $b_{j}$ are the elementary symmetric functions of $(a)$ to within a constant factor and are called the mixed volumes. It is easy to check that the concavity result of Fenchel ${ }^{2}$ reduces in this case to the statement that $E_{r}{ }^{1 / r}(a)$ is concave.

3. Applications to Matrices. The following result is known $(3 ; 4)$ : Let

$$
f\left(x_{1}, \ldots, x_{k}\right)=E_{r}\left(\left(A x_{1}, x_{1}\right), \ldots,\left(A x_{k}, x_{k}\right)\right),
$$

where $A$ is a n.n.h. matrix with characteristic roots $0 \leqslant \alpha_{i} \leqslant \alpha_{i+1}(i=1, \ldots$, $n-1)$. Then

$$
\begin{aligned}
& \max f=\left(\begin{array}{l}
k \\
r
\end{array}\right)\left(\sum_{j=1}^{k} \frac{\alpha_{n-j+1}}{k}\right)^{r} \\
& \min f=E_{r}\left(\alpha_{1}, \ldots, \alpha_{k}\right)
\end{aligned}
$$

where both max and min are taken over all sets of $k$ o.n. vectors in the unitary n-space. ${ }^{3}$

${ }^{2} \mathrm{~A}$ discussion of mixed volumes and a conjecture of this general concavity result may be found in the book by T. Bonnesen and W. Fenchel, Theorie der konvexen Körper (Chelsea, New York, 1948), p. 93.

${ }^{3}$ The paper of Ostrowski contains only the value of $\min f$. 
Theorem 3. Let $A, B, A+B$ be n.n.h. with characteristic roots $0 \leqslant \alpha_{i} \leqslant \alpha_{i+1}$, $0 \leqslant \beta_{i} \leqslant \beta_{i+1}, \quad 0 \leqslant \lambda_{i} \leqslant \lambda_{i+1}$ for $i=1, \ldots, n-1$, respectively. Then for $1 \leqslant r \leqslant k \leqslant n$

$$
E_{r}^{1 / r}\left(\lambda_{1}, \ldots, \lambda_{k}\right) \geqslant E_{r}^{1 / r}\left(\alpha_{1}, \ldots, \alpha_{k}\right)+E_{r}^{1 / r}\left(\beta_{1}, \ldots, \beta_{k}\right) .
$$

Proof. Let $x_{1}, \ldots, x_{k}$ be an o.n. set of characteristic vectors of $A+B$ such that

$$
c_{j}=\left((A+B) x_{j}, x_{j}\right)=\lambda_{j} \quad(j=1, \ldots, k) .
$$

Set

$$
\left(A x_{j}, x_{j}\right)=a_{j}, \quad\left(B x_{j}, x_{j}\right)=b_{j}
$$

Then

$$
\begin{aligned}
E_{r}^{1 / r}(\lambda) & =E_{r}^{1 / r}(c)=E_{r}^{1 / r}(a+b) \\
& \geqslant E_{r}^{1 / r}(a)+E_{r}^{1 / r}(b) \geqslant E_{r}^{1 / r}(\alpha)+E_{r}^{1 / r}(\beta)
\end{aligned}
$$

Let

$$
x^{n}+\sum_{j=1}^{n} \rho_{j}(A) x^{n-j}
$$

be the characteristic polynomial of $A$. By setting $k=n$ in Theorem 3 we obtain the following extension of the Minkowski inequality to all the coefficients in the characteristic polynomial:

THEOREM 4. If $A$ and $B$ are n.n.h. then for $1 \leqslant r \leqslant n$

$$
\left|\rho_{r}(A+B)\right|^{1 / r} \geqslant\left|\rho_{r}(A)\right|^{1 / r}+\left|\rho_{r}(B)\right|^{1 / r} .
$$

The extension of Fan's result is contained in

Theorem 5. If $A$ and $B$ are n.n.h. and $\omega+\sigma=1, \omega \geqslant 0, \sigma \geqslant 0$, then for $1 \leqslant r \leqslant n$

$$
\left|\rho_{r}(\omega A+\sigma B)\right| \geqslant\left|\rho_{r}(A)\right|^{\omega}\left|\rho_{r}(B)\right|^{\sigma} .
$$

Proof. As in the proof of Theorem 3

$$
\begin{aligned}
E_{r}^{1 / r}(\lambda) & =E_{r}^{1 / r}(\omega(a)+\sigma(b)) \geqslant \omega E_{r}^{1 / r}(a)+\sigma E_{r}^{1 / r}(b) \\
& \geqslant \omega E_{r}^{1 / r}(\alpha)+\sigma E_{r}^{1 / r}(\beta) \geqslant E_{r}^{\omega / r}(\alpha) E_{r}^{\sigma / r}(\beta) .
\end{aligned}
$$

The result follows by taking $r$ th powers.

THEOREM 6. Under the same hypotheses as Theorem 3,

$$
\begin{aligned}
& k^{1-r}\left(\begin{array}{c}
k \\
r-1
\end{array}\right) \mathbf{E}_{r}\left(\lambda_{1}, \ldots, \lambda_{k}\right) \\
& \geqslant E_{r}\left(\alpha_{1}, \ldots, \alpha_{k}\right)\left(\sum_{j=1}^{k} \alpha_{n-j+1}\right)^{1-r} \\
& \quad+E_{r}\left(\beta_{1}, \ldots, \beta_{k}\right)\left(\sum_{j=1}^{k} \beta_{n-j+1}\right)^{1-r} .
\end{aligned}
$$


Proof. We again choose $x_{1}, \ldots, x_{k}$ to be $k$ o.n. characteristic vectors of $A+B$ corresponding respectively to the characteristic roots $\lambda_{1}, \ldots, \lambda_{k}$. Then

$$
\left((A+B) x_{j}, x_{j}\right)=\lambda_{j}, \quad j=1, \ldots, k
$$

and we have

$$
\begin{aligned}
\mathbf{E}_{r}\left(\lambda_{1}, \ldots, \lambda_{k}\right) & =\mathbf{E}_{r}(((A+B) x, x))=\mathbf{E}_{r}((A x, x)+(B x, x)) \\
& \geqslant \mathbf{E}_{r}((A x, x))+\mathbf{E}_{r}((B x, x)) \\
& \geqslant E_{r}(\alpha)\left(\begin{array}{c}
k \\
r-1
\end{array}\right)^{-1}\left(\sum_{j=1}^{k} \frac{\alpha_{n-j+1}}{k}\right)^{1-r} \\
& +E_{r}(\beta)\left(\begin{array}{c}
k \\
r-1
\end{array}\right)^{-1}\left(\sum_{j=1}^{k} \frac{\beta_{n-j+1}}{k}\right)^{1-r},
\end{aligned}
$$

and (9) follows.

COROLlary 1. Under the same hypotheses as Theorem 4

$$
n^{1-r}\left(\begin{array}{c}
n \\
r-1
\end{array}\right)\left|\frac{\rho_{r}(A+B)}{\rho_{r-1}(A+B)}\right| \geqslant \frac{\left|\rho_{r}(A)\right|}{(\operatorname{tr} A)^{r-1}}+\frac{\left|\rho_{r}(B)\right|}{(\operatorname{tr} B)^{r-1}} .
$$

Proof. Set $k=n$ in (9). We immediately conclude from Corollary 1 that

$$
\operatorname{det}(A+B) \geqslant \operatorname{tr}(A+B)\left(n^{-\frac{1}{2} n(n-1)} \prod_{r=2}^{n}\left(\begin{array}{c}
n \\
r-1
\end{array}\right)^{-1} \sigma_{r}\right)
$$

where

$$
\sigma_{r}=\frac{\left|\rho_{r}(A)\right|}{(\operatorname{tr} A)^{r-1}}+\frac{\left|\rho_{r}(B)\right|}{(\operatorname{tr} B)^{r-1}}
$$

\section{REFERENCES}

1. K. Fan, On a Theorem of Weyl concerning eigenvalues of linear transformations II, Proc. Nat. Acad. Sci., 36 (1950), 31.

2. W. Fenchel, Généralisation du théorème de Brunn-Minkowski concernant les corps convexes, C.R. des Sci. de l'Acad. des Sci., 203 (1936), 764-766.

3. M. Marcus and J. L. McGregor, Extremal properties of Hermitian matrices, Can. J. Math., 8 (1956), 524-531.

4. A. Ostrowski, Sur quelques applications des fonctions convexes et concaves au sens de I. Schur, J. Math. pures et appl. (9), $\$ 1$ (1952), 253-292.

University of British Columbia

United States Naval Ordnance Test Station

Pasadena, Cal. 